\title{
The Effect of Glucagon-like Peptide-1 (GLP-1) on Obesity
}

\author{
Xiaoyi Huang, Meihua Liang and Biyue Wei* \\ Biotherapy Center, Tumor Hospital of Harbin Medical University, Harbin, 150081, China
}

${ }^{*}$ Corresponding author: Biyue Wei, Biotherapy Center, Tumor Hospital of Harbin Medical University, Harbin, 150081, China, E-mail: lihuaxiaomiao@163.com

Received date: November 06, 2018; Accepted date: November 23, 2018; Published date: November 29, 2018

Copyright: (C) 2018 Huang X, et al. This is an open-access article distributed under the terms of the Creative Commons Attribution License, which permits unrestricted use, distribution, and reproduction in any medium, provided the original author and source are credited.

Citation: Huang X, Liang M, Wei B (2018) The Effect of Glucagon-like Peptide-1 (GLP-1) on Obesity. Crit Care Obst Gyne Vol.5 No.1:4.

\section{Abstract}

Obesity has become a major topic of medical research in the world. In addition to diet control, exercising and behavioral therapy, many scholars now believe that drug therapy should be added to the routine treatment of obesity. It is confirmed that weight-loss drugs can help improve the health of obese patients, and patients who fail to intervene in lifestyle alone can benefit from drug treatment. Glucagon-like peptide-1 (GLP-1) is currently used in clinical blood glucose control drugs, but it has been proposed that taking GLP-1 and GLP-1 analogs in obese patients can not only achieve weight loss but also prevent complications.

Keywords: Obesity; Glucagon-like peptide-1; Coronary heart disease; Endocrinology

\section{Abbreviations:}

GLP-1: Glucagon-Like Peptide-1; El: Energy Intake; WHO: World Health Organization; BMI: Body Mass Index; FDA: Food and Drug Administration; WC: Waist Circumference; EE: Energy Expenditure; CNS: Central Nervous System; REE: Resting Energy Expenditure; CVD: Cardiovascular Disease

\section{Introduction}

Obesity has become a major topic of medical research in the world. In June 2013, the American medical association first declared obesity a disease. The disease in which no obvious endocrine, metabolic disease, and another pathogen can be found is called simple obesity. Simple obesity is a nutritional disorder disease in which the long-term Energy Intake (EI) exceeds the consumption of the human body, resulting in excessive accumulation of fat and excess weight. It is a serious, chronic, relapsing disease of energy regulation, with strong genetic and early-life environmental determinants [1]. According to the World Health Organization (WHO), Body Mass Index (BMI) greater than $25 \mathrm{~kg} / \mathrm{m}^{2}$ is overweight, BMI greater than 30 $\mathrm{kg} / \mathrm{m}^{2}$ is obese [2]. According to the data collected and analyzed by the China obesity task force, the $\mathrm{BMl}>24 \mathrm{~kg} / \mathrm{m}^{2}$ is overweight and the $\mathrm{BMI}>28 \mathrm{~kg} / \mathrm{m}^{2}$ is obese [3]. Obesity is a chronic metabolic disease caused by many factors and is a risk factor for many diseases, such as hypertension, coronary heart disease, ischemic stroke, diabetes and gout [4,5]. At the same time, obese patients are more likely to have mobility difficulties, accompanied by joint pain, swelling, muscle soreness, and other uncomfortable symptoms. Physical discomfort reduces the number of activities of obese people, causing fear of activities and affecting normal social functions. However, impaired social function and mainstream aesthetic criticism make them prone to low self-esteem, anxiety and other adverse psychological emotions, thus affecting their mental health $[6,7]$. Obesity is now the most prevalent chronic disease in the United States, which amounts to an estimated \$147 billion in health care spending annually. Moreover, most obese people lose weight for a small period and then they regain all the weight that they have lost or even worse, they increase their weight more than before starting a diet [8]. The long-term goal of obesity treatment is to reduce body weight by $5-10 \%$, maintain a $B M \mathrm{~K}<25 \mathrm{~kg} / \mathrm{m}^{2}$, lower blood pressure, blood glucose, and lipid levels, and other risk factors [9]. Current obesity treatment measures include:

\section{Lifestyle intervention}

\section{Drug intervention}

\section{Surgical treatment [10]}

Although lifestyle modification is considered first-line treatment, it is often ineffective, difficult, especially in the long term [11-13]. For surgery, it has strict operation indications:

\section{Patients with severe obesity (BMI) of $40 \mathrm{~kg} / \mathrm{m}^{2}$}

\section{BMI of $35^{\sim} 40 \mathrm{~kg} / \mathrm{m}^{2}$, but have related complications}

\section{Behavioral therapy and drug treatment are invalid [14]}

Therefore, surgery cannot adapt to all patients, and surgery has trauma and corresponding risks. Therefore, appropriate drug intervention has become the focus of current research. In 2015, the American Society of Endocrinology, in collaboration with the European association and the obesity association, jointly published the "Obesity drug management: clinical practice guidelines of the American Society of Endocrinology". It is confirmed that weight-loss drugs can help improve the health of obese patients, and patients who fail to intervene in lifestyle alone can benefit from drug treatment [15]. The drugs may be 
significantly beneficial to patients in need of weight loss as they are comparably effective for intensive programs to promote changes in eating habits and lifestyles $[16,17]$.

\section{Current application of weight loss drugs}

There are two types of drugs that can be used according to the time of approval by the Food and Drug Administration (FDA). Drugs that can be used for more than 12 weeks ( 3 months) are long-term therapeutic drugs. Because of the lack of safety and efficacy data for long-term use, those approved for use less than 12 weeks are short-term treatment drugs.

Short-term therapeutic drugs: Short-term therapeutic drugs are drugs that lack long-term safety data and are approved by the FDA for a period of up to 12 weeks. Obesity is a chronic disease, needing management for a long time. The weight is very easy to rebound after the drugs were stopped. At the same time, because of the abuse of possibility and common adverse reactions (such as central nervous excitement, high blood pressure, and heart rate, etc.), the short-term use of drugs in Europe was withdrawn en masse. The four short-term drugs currently approved are epinephrine appetite suppressants [18].

Phentermine: Phentermine, an epinephrine appetite suppressant, is a central appetite suppressant, which can stimulate the sympathetic nervous system to release norepinephrine. Phentermine affects the brain's dopamine, 5the neurotransmitters such as serotonin, ammonia, increased heart rate, blood pressure, reduce appetite $[19,20]$. Phentermine was approved for sale in 1959 , with $30-37.5 \mathrm{mg} / \mathrm{d}$, making it the most prescribed obesity treatment in the United States. What is more, Phentermine is also effective for weight reduction in a pediatric weight management clinic [21]. Kim Kyoung Kon, conducted a randomized, double-blind, placebocontrolled study had been performed between February and July 2005, in Seoul on 68 relatively healthy obese adults whose BMI was $25 \mathrm{~kg} / \mathrm{m}^{2}$ or greater [22]. They received Phentermine$\mathrm{HCl} 37.5 \mathrm{mg}$ or placebo once daily with behavioral therapy for obesity. Circumference in Phentermine-treated subjects was significantly greater than that of the placebo group (weight: -6.7 $\pm 2.5 \mathrm{~kg}, \mathrm{p}<0.001$; Waist Circumference (WC): $-6.2 \pm 3.5 \mathrm{~cm}$, $\mathrm{p}<0.001)$. Dry mouth and insomnia were the only statistically significant adverse events that occurred more frequently in Phentermine group. Most side effects of Phentermine were mild to moderate in intensity. Short-term Phentermine administration induced significant weight reduction and reduction of WC without clinically problematic adverse events on relatively healthy Korean obese people. Phentermine has a lower risk of pulmonary hypertension than fenfluramine, which has been withdrawn from the market [23].

Amfepramone: Amfepramone, an epinephrine appetite suppressant, the main mechanism of action is similar to that of fentamine. It can excite the satiety center on the ventral side of the hypothalamus, and produce the feeling of satiety [24]. Amfepramone was approved for sale in 1959; fast release tablets can be used by $25 \mathrm{mg}$, 3 times a day and slow release tablets can be used by $75 \mathrm{mg}, 1$ time a day. The adverse reactions are similar to Phentermine.
Benzylamine: Benzylamine, an epinephrine appetite suppressant, was approved for sale in 1960, with 25-50 mg TID. It is a Schedule III anorectic agent [25]. The adverse reactions are similar to Phentermine.

Phendimetrazine: Phendimetrazine, an epinephrine appetite suppressant, was approved for sale in 1959, with $17.5-70 \mathrm{mg}$ TID. The adverse reactions are similar to Phentermine. Cho, et al. has reported a case in which central retinal vein occlusion noted 2 days after use of Phendimetrazine as an appetite suppressant [26].

\section{Long-term therapeutic drugs:}

Orlistat: Orlistat is currently the only non-central action drug, namely gastrointestinal lipase inhibitor, which was approved for market in 1998 and entered China in October 2000. With $120 \mathrm{mg}$ TID. Orlistat, through competitive with stomach lipase inside the stomach, small bowel antrum and pancreatic lipase, the deactivation of the enzyme in the food cannot be mainly triglyceride hydrolysis of fats to absorbable free fatty acids and single acylglycerol, undigested triglycerides do not get absorbed by the body, so as to reduce calorie intake, achieve the purpose of weight control $[27,28]$.

The main adverse reactions are gastrointestinal and include diarrhea, fecal incontinence, anal oil, flatulence, and indigestion. These adverse reactions are more apparent when eating a highfat diet. Because of these adverse reactions, Orlistat may not have good compliance [29]. On July 8, 2010, the FDA issued Orlistat or cause liver damage the security of information, prompt a few patients taking diet pill Orlistat severe liver damage, but using Orlistat and the specific relationship between the cause serious liver damage is uncertain. The accompanying symptoms of liver damage include pruritus, yellowing of the skin or eyes, blackened urine, loss of appetite, or lightened stool color. Sall, et al. reported on a 54-year-old African-American woman with high blood pressure and liver failure. She developed increasing fatigue, jaundice and confusion [30]. She has been taking the over-the-counter Orlistat for the past two months. Physical examination revealed sclera yellow, jaundice, and instability and speech retardation. Laboratory tests showed significant abnormalities in coagulation. Acute virus and autoimmune serum are negative for toxicological screening. Liver biopsy revealed hepatic parenchymal necrosis, possibly secondary to drug toxicity. Based on her clinical presentation and time history, the liver injury patterns seen in liver biopsies, and the lack of other reasonable explanations, her liver failure is likely to be related to Orlistat use. Her condition continued to deteriorate and she eventually received an orthotopic liver transplant. In addition, he reported 14 cases of severe liver damage associated with Orlistat.

Lorcaserin: Lorcaserin is an optional $5-\mathrm{HT} 2 \mathrm{C}$ receptor agonist. It has 15 and 100 times the affinity of 5-HT2A and 5-HT2B receptors, respectively. Lorcaserin was approved for sale in June 2012, with $10 \mathrm{mg}$ BID. Lorcaserin lost weight by reducing $\mathrm{EI}$ without changing Energy Expenditure (EE) or Respiratory Quotient (RQ) [31].

The manufacturer recommends that patients taking other serotonergic drugs such as antidepressants avoid co- 
administration of Lorcaserin. Psychiatric symptoms including euphoria, hallucination, and dissociation rarely occur with recommended dosages, but these have occurred in 19\% of patients taking 40-60 mg daily. Lorcaserin does not increase suicidal thoughts or actions and does not cause mood changes or trouble sleeping when used as recommended [32]. The 5HT2C receptor was found only in the Central Nervous System (CNS), avoiding the activation of the 5-ht2b receptor on the heart valve, thereby avoiding the heart valve injury [33]. However, patients with congestive heart failure should be cautious about the use of Lorcaserin. In addition, attention should be paid to the potential risk of addiction. Still, have a few patients can appear giddy, headache.

Naltrexone-bupropion: Naltrexone-bupropion was approved by the FDA on September 10, 2014. Naltrexone is opioid receptor antagonist hydrochloric acid, bupropion is an antidepressant drugs amino ketones. They act on different regions of the brain that regulate food intake: the hypothalamus (center for appetite regulation) and the centre of the dopamine circuit (reward system). The most common adverse reactions in the research of Ning, et al. are nausea (6.3\%), headache (1.7\%) and vomiting (1.1\%) [32].

\section{Glucagon-like peptide-1 (GLP-1)}

GLP-1 is synthesized from duodenum, $L$ cells of the small intestine and large intestine, a small number of synthesized from the pancreas and the hypothalamus, the nutrition and under the action of a nerve, endocrine factors stimulate secretion of a multifunctional peptide hormone, including regulating blood sugar. GLP-1 secretion in the gastrointestinal tract is affected by glucose and fatty acid levels in the blood after food digestion and absorption, and vagus nerve stimulation also affects its secretion process [34]. Current studies confirm that GLP-1 can reduce appetite and food intake, reduce gastrointestinal motility, delay gastric emptying, and reduce weight [35-37].

CNS: GLP-1 mainly effects on GLP-1 receptors in hypothalamus paraventricular nucleus, mesencephalic limbic system, and posterior brain, delaying and reducing animal feeding. GLP-1 can cause the central system to produce a short period of satiety and loss of appetite. Kanoski, et al. also demonstrated that GLP-1 inhibits appetite through the CNS's GLP-1 receptor, slowing down gastric emptying and thus slowing down the rate at which food is absorbed into the bloodstream [38]. Food intake suppression is mediated by activation of GLP-1R expressed on vagal afferents as well as direct CNS GLP-1R activation [39].

Gastrointestinal tract: In the gastrointestinal tract, GLP-1 can inhibit gastric emptying, inhibit the contraction of the pylorusduodenal segment, and secrete gastrin and dietary acid [40]. GLP-1 is produced by food stimulation and acts on the GLP-1 receptor in the gastrointestinal tract, which has the effect of delaying gastric emptying and nutrient absorption. Some studies have shown that GLP-1 has a significant effect on gastric emptying deceleration. Gastric emptying coefficients were significantly reduced with increasing doses of GLP-1. Gastric emptying was decelerated by GLP-1 in a dose-dependent fashion. It might, therefore, be reasonable to assess gastric emptying before initiating GLP-1 treatment to avoid the induction of gastroparesis in these patients [41].

Energy consumption: Some energy consumption studies have shown that GLP-1 can increase But Maciel, et al. think that GLP-1 has no short-term effect on Resting Energy Expenditure (REE) but may decrease Diet-Induced Thermogenesis (DIT). The GLP-1RA Exenatide and Liraglutide had a neutral effect on REE, although it was not possible to rule out an increase in REE following prolonged treatment [42].

Lipid: Lilalutin can significantly reduce TC, TG and LDL-C levels and reduce body mass in the serum of hyperlipidemic mice, and some studies have shown that this decrease is mainly manifested in subcutaneous and visceral fat. ShaSha Chowdhury Sumon Rahman, the experimental results show that a high-fat diet induced mice were randomly divided into the lalu peptide group, the dietary intervention group and continue to high-fat feeding group, the lalu peptide group total fat content, mesenteric fat, epididymal fat, kidney week fat, fat and subcutaneous fat under the shoulder blade down more obvious compared with the other groups $[43,44]$

\section{Analogs of GLP-1}

GLP-1 has a good effect on obese patients. However, GLP-1 is unstable in the body, easy to be degraded by Dipeptide Base Peptidase IV (DPP IV) rapidly and quickly removed by the kidney. Its half-life is very short, about 1.5-2.0 minutes. In the plasma of healthy people and patients with T2D, only $1 / 3 \sim 1 / 2$ of GLP-1 are active GLP-1, and the rest are inactive fragments [45]. Therefore, long-term GLP-1 and its analogs have become one of the research hotspots. Current results suggest that GLP-1 has a positive short-term utility, but a short half-life and a short action time. High bioavailability of GLP-1 analogs will benefit the clinical treatment of obesity.

Liraglutide: Liraglutide was in GLP-1 (7-37) based on modified products, is a long-acting GLP-1 analog, which is endogenous GLP-1 $26^{\text {th }}$ amino acids to increase a 16 carbon palmitoyl fatty acid side chain and to become replacement of the $34^{\text {th }}$ lysine arginine after modification of the peptides, and natural person GLP-1 has $97 \%$ homology, relative molecular mass is 3751.2 , the molecular structure characteristics make it can be avoided by DPP-4 enzyme hydrolysis, half-life reached around $13 \mathrm{hrs}$ [46]. The drug was approved internationally as a weight-loss drug in 2014. It can be subcutaneously injected by $0.6 \mathrm{mg}$ per day. Comparative data suggest that weight loss with Liraglutide is greater than that seen with Orlistat or Lorcaserin, but slightly less than seen with Phentermine/Topiramate. It is found in the clinical trial by Mehta, et al. [47].

It's worth emphasizing that pathogenic mutations in the hypothalamic appetite-regulating Melanocortin-4 Receptor (MC4R) are the most common cause of monogenic juvenileonset obesity with a global prevalence of up to $6 \%$. However, the experiment proved that the weight-reducing and glucoselowering effects of the GLP-1RA Liraglutide are preserved in spite of defective MC4R activity in patients with obesity caused by MC4R mutations [48]. 
An experiment has proved that the weight effect of rilalupe has been maintained for at least 12 months in patients with diabetes for a long time [49]. This suggests that GLP-1 analogs have a reliable long-term effect on obesity. However, other studies suggested that the use of Liraglutide may be harmful in patients with severe heart failure, in part due to an increase in heart rate. Thus, patient except for patients with severe heart failure can accept the treatment. Further studies are needed to evaluate the long-term effects of Liraglutide [50]. The cardiovascular safety of Liraglutide, a GLP-1RA approved for weight management at a dose of $3.0 \mathrm{mg}$, was evaluated post hoc using data from 5908 participants in 5 randomized, doubleblind, placebo-controlled clinical trials. In this analysis, Liraglutide $3.0 \mathrm{mg}$ treatment was not associated with excess cardiovascular risk [51,52].

A possible association between GLP-1 analogs and incidences of pancreatitis has been suggested based on clinical studies. In overtly diabetic male and female ZDF rats, prolonged exposure to GLP-1 receptor agonists does not affect biochemical or histopathological markers of pancreatitis, and whereas both Exenatide and Liraglutide increase $\beta$-cell mass, they have no effect on the exocrine pancreas. Howeverstudies in human is needed to explain the relationship [53].

Exenatide: Unlike GLP-1, the n-terminal and c-terminal of Exenatide are not $\alpha$-spirals, and the $n$-terminal is an irregular curl. C-terminal is an irregular hydrophilic structure called "trpcage", which is called tryptophan Cage. The amino acid residues in the $7^{\text {th }} 28^{\text {th }}$ positions formed the $\alpha$-spiral [54]. The half-life of twice-daily Exenatide is 2.4 hours. Exenatide is first GLP-1 analog approved for sale by the U.S. FDA in 2005. It was approved for listing in China in 2009 and is the first GLP-1 analog to be listed in China. In week 1 to 4 , Exenatide can be injected subcutaneously by $5 \mu \mathrm{g} 60$ minutes before breakfast and dinner, and in week 5 to $12,10 \mu \mathrm{g}$ is injected subcutaneously within 60 minutes before breakfast and dinner. It is used for weight loss and blood glucose control in obese patients with diabetes mellitus. This effect was dose-dependent and influenced by food carbohydrate content, but not by the lag time between Exenatide injection and meal ingestion [55]. It is reported that Exenatide treatment was no less effective than metformin in improving endothelial function [56]. However, with Exenatide twice daily, antibody formation against the compounds is frequent being more antigenic [57]. The most common adverse reactions of GLP-1 agonists are gastrointestinal reactions such as nausea and vomiting. Among them, long-acting GLP-1 receptor agonist has less effect on gastric emptying due to its small effect on gastric emptying. Moreover, the body ADAPTS rapidly under continuous GLP-1 stimulation, so its gastrointestinal reaction is less severe and lasts a little bit longer [58]. Exenatide significantly improved glucose control and decreased body weight, without increased hypoglycemia or unexpected safety findings [59].

Semaglutide: Semaglutide were modified in the $8^{\text {th }}$ position as $\alpha$-aminoisobutyric acid, and the lysine in the $26^{\text {th }}$ position of the peptide chain was connected to the side chain of 18 carbon adipose acids, while the lysine in the $34^{\text {th }}$ position was replaced by arginine. It has $94 \%$ homology with GLP-1 in human. On
December 5, 2017, the FDA approved the release of Semaglutide. The initial dose of Semaglutide was $0.25 \mathrm{mg}$, subcutaneous injection once a week for 4 consecutive weeks. Thereafter, the dose increased to $0.5 \mathrm{mg}$ per dose. If blood sugar is not well controlled, the dose of $0.5 \mathrm{mg}$ will increase to as much as $1 \mathrm{mg}$ per session once a week after 4 weeks of maintenance. It is reported that Semaglutide-induced weight loss was consistently greater versus comparators, regardless of baseline BMI [60]. In one experiment, for diabetics, Semaglutide increased weight loss more than weekly Exenatide ER [61]. Semaglutide can significantly reduce $\mathrm{HbA} 1 \mathrm{c}$, body weight and systolic blood pressure [62].

Semaglutide demonstrated improved glycaemic control and decreased body weight with a safety profile similar to other GLP-1 Receptor Agonists (GLP-1RAs) [63]. Nevertheless, we noted an increased incidence of nausea, vomiting, and diarrhea. Semaglutide significantly reduces systolic blood pressure. However, it is associated with an increased incidence of gastrointestinal adverse events. Results for pancreatitis and retinopathy require further assessment in post-approval pharmacovigilance studies [62]. One study showed that Semaglutide did not increase the additional risk for older patients [64].

Lixisenatide: The structure of Lixisenatide has a deletion of proline and an addition of six lysine amino acids at the carboxyl terminus in order to stabilize the peptide in circulation. The resulting half-life for Lixisenatide is 3 hours. Lixisenatide received FDA approval on July 28, 2016. In the first 14 days, $10 \mu \mathrm{g}$ were injected subcutaneously once a day. The dose increased to $20 \mathrm{~g}$ once a day for 15 days later. Lixisenatide protects against cerebral ischemia/reperfusion injury in diabetic rats. Lixisenatide relieved carotid endothelial dysfunction by increasing endothelial Nitric Oxide Synthase (eNOS) expression. It also dampened vascular nitrosative/oxidative stress via suppression of iNOS and NADPH oxidase expressions [65]. However, Lixisenatide was inferior with regard to body weight loss compared with Exenatide [66]. At the same time, the effect of Lixisenatide on fasting plasma levels of glucose and $\mathrm{HbAlc}$ is inferior to that of the long-acting compounds [67]. Nausea is the most frequent adverse event. Nausea events were generally of mild-to-moderate intensity and were reported more frequently during the first 3 weeks of treatment, with a reduced occurrence from week 7 to the end of treatment [68]. On constipation, Kapitza, et al. indicated a prevalence of less than 6\% [69]. The same to other GLP-1, use with insulin or sulfonylurea, Lixisenatide may cause hypoglycemia Monami, et al. believe that the incidence of pancreatitis and pancreatic cancer with GLP-1RA was not significantly different from that observed in comparator arms [70]. But at the same time, Chis, et al. reported a case of acute pancreatitis during GLP-1 receptor agonist treatment [71]. In the animal, experimental studies pancreas related acute or chronic diseases were also described as sideeffects of GLP-1RA [72].

A large dosage of Lilaludin (Exenadine slow-release injection suspension): On January 27, 2012, Bydureon was approved by FDA. As a new formulation of once-weekly Exenatide, it has been developed by incorporating the active molecule into a 
biodegradable polymeric microsphere, allowing gradual drug delivery over an extended period [73]. It can be subcutaneously injected by $2 \mathrm{mg}$ per time, 1 time per week.

It improves patient compliance and has good control of fasting blood glucose and glycosylated hemoglobin. The intensity of glycaemic control with Exenatide ER was generally better than that observed with the Exenatide immediate-release formulation (twice daily), Sitagliptin or insulin glargine [74]. Other side effects include nodules under the skin, swelling, and itching at the injection site. This was a little more serious than which in the immediate release of Exenatide.

\section{Complications of obesity}

Hypertension, coronary heart disease and other cardiovascular diseases (CVDs): CVD is a major health hazard. The study found that BMI and WC were independently and positively associated with clustering rate of other risk factors for CVD. It is very important for health to keep both BMI and WC in normal level [75]. Aditya Goud, et al. believed that GLP-1 also increased the excretion of $\mathrm{Na}^{+}$through the Anp- $\mathrm{Na}^{+} / \mathrm{H}^{+}$ exchange pathway of proximal tubule of the kidney. The release of endothelial NO or non-dependent cGMP by akt-eNOS pathway makes vascular smooth muscle diastole play a role in lowering blood pressure [76].

Blood pressure: GLP-1 can relax blood vessels and reduce blood pressure by binding to GLP-1 receptors on the surface of the atrium. Kim, et al. confirmed that GLP-1RA indirectly plays the role of hypotension by stimulating the release of atrial natriuretic peptide [77].

Atherosclerosis: GLP-1 by improving endothelial function, reduce hemal wall adhesion and inflammatory cells infiltration, inhibit intimal thickening, indirectly improve glucolipid metabolism, so as to play against atherosclerosis (atherosclerosis, AS), and cardiovascular protection.

Other results suggest that Exenatide exerts significant cardioprotective effects against oxidative stress-induced injury in vitro and in vivo. The mechanisms involved may be attributed to the scavenging of oxidative stress products, such as ROS, the increase in the concentrations of antioxidant defense enzymes and the inhibition of cardiomyocyte apoptosis. The anti-apoptotic effects of Exenatide were, at least in part, associated with the activation of the PI3K/Akt signaling pathway [78]. GLP-1R has been localized to mouse aortic smooth muscle and endothelial cells, as well as monocytes and macrophages, using immunocytochemistry and Western blotting (140). GLP-1 can reduce monocyte adhesion to aortic endothelial cells, associated with a reduction in atherosclerotic lesion size. GLP-1 reduced neointimal formation in response to endothelial denudation of the femoral artery and may have reduced foam cell formation in macrophages [79]. Liraglutide can also directly protect cardiomyocytes from reperfusion injury and reduce myocardial injury after myocardial infarction, possibly by regulating intracellular calcium homeostasis [76]. In a study, Tomohiko Kimura found the down-regulation of vascular GLP-1 receptor expression in human subjects with obesity and they believed that the decrement of vascular GLP-1 receptor expression was involved in the progression of arteriosclerosis and the onset of cardiovascular events [80]. While the analogs of GLP-1 is able to improve the situation. All of these directly reduce the incidence of heart disease. GLP-1 can also indirectly reduce the risk of CVD by lowering blood pressure, reducing body mass and improving blood lipid.

Fatty liver disease: Due to insulin resistance, obese patients often have abnormal lipid metabolism: high TG, Very LowDensity Lipoprotein (VLDL) and Low-Density Lipoprotein (LDL), leading to fatty liver.

Some studies have shown that GLP-1 may significantly reduce triglycerides and cholesterol levels and reduce the synthesis of low-density lipoprotein by increasing the expression of hepatic ApoE protein or increasing the adiponectin level. The protective effect of GLP-1 on hepatic fatty changes can also be seen in dietinduced obese rats [81].

Furthermore, in a study comparing 26 weeks of treatment with Liraglutide, $3.0 \mathrm{mg}$ once daily to lifestyle intervention (the currently recommended treatment), patients achieved similar reductions in levels of alanine aminotransferase, liver fat fraction, liver stiffness and body weight [82]. Kirstine, et al. have shown that Liraglutide reduced liver weight. Biochemical (plasma, liver) and quantitative histological (liver) analyses were applied. Liraglutide reduced total liver TG and TC content and significantly reduced plasma ALT and AST [49].

Immune function: The GLP-1 analog clinical efficacy can activate iNKT cells, which can induce FGF21 to lose weight in both humans and mice. These findings reveal an iNKT cell-FGF21 axis that defines a new immune-mediated pathway that could be targeted for weight regulation [83].

Recent studies have shown that obesity can affect immune function. Fat cells have the function of macrophage sample before, it may be involved in inflammation, can also affect the body's nonspecific immunity and nonspecific immune, humoral immunity and cellular immune function change, so that the chance of bacterial infections and more serious [84]. Liraglutide exerts marked anti-oxidative and anti-inflammatory effects on endothelial cells with inhibition of PKC- $\alpha$, NADPH oxidase, NF-KB signaling and up-regulation of protective anti-oxidative enzymes [85]. When patients in critical conditions, often accompanied by persistent high blood glucose and immune dysfunction, the hypoglycemic advantages of GLP-1 and its immune regulating effect on immune cells, it is the key node of critically ill patients [86].

Metabolic syndrome: Exenadine can stimulate insulin secretion and inhibit the improper decomposition of glycogen by means of glucose dependence, delay gastric emptying and increase the sense of satiety. Exenatide can significantly reduce average weight and BMI. The weight loss and average abdominal circumference of $76.6 \%$ of patients were significantly reduced. Further analysis revealed that average triglycerides, total cholesterol, systolic and diastolic blood pressure all decreased significantly. In an experimentExenatide treatment was associated with significant reductions in mean body weight and BMI. Weight loss in $76.6 \%$ of patients was concomitant with a significant reduction in mean abdominal girth. Further analysis 
revealed significant decreases in mean triglycerides, total cholesterol, and both systolic and diastolic blood pressure [87].

\section{Discussion}

Obesity has become an important subject of medical research in the world. In physiological it may be the risk factors for disease such as high blood pressure, coronary heart disease, diabetes, and other diseases. At the same time, it may cause inferiority, anxiety and another adverse psychological mood, which affects the mental health. The social and psychological problems associated with obesity may depend on the cultural background rather than how much is overweight. Obesity is on the rise in China. It is important to understand the influence of obesity on mental behavior and to strengthen the control rate of obesity. There are many treatments for obesity, the most effective being surgery. Due to the indications and vice damage, many patients refuse the therapy but in the face of lifestyle and diet control invalid should timely give drug intervention, prevent to produce more complications, patients health threat. However, in the face of ineffective lifestyle and diet control, drug intervention should be timely given to prevent more complications and threaten the health of patients. Currently, there are many kinds of weight loss drugs in clinical application, among which short-effect drugs cannot be used for a long time due to the lack of long-term safety data. But obesity is a chronic disease, and most of it will rebind after drug intervention is stopped. However, the choice of long-acting therapeutic drugs is relatively limited, and the most commonly used gastrointestinal lipase inhibitor, Orlistat, has not been clearly explained in relation to severe liver injury. GLP-1 has attracted much attention from the medical community as an emerging drug due to its excellent function on hypoglycemic affection. We found that besides its hypoglycemic affection, it also had a good effect on the weight loss so that obese patients could benefit from it whether they had diabetes or not.

GLP-1 has a short survival time in the physiological state, with a half-life of only 1.5-2.0 minutes. Therefore, the more stable GLP-1 analog with similar physiological effects has become a hot research topic.

The first to be approved for sale is Exenatide, which is generally used as an indication of diabetes. In patients with type 2 diabetes mellitus with obesity, several trials have shown a statistically significant difference in weight loss from placebo. Exenatide is currently administered by injection. Due to its short half-life, it needs to be given twice a day. Studies have reported that Exenatide is made into enteric-soluble oral preparation, and the capsule remains intact in the stomach [88]. After arriving in the small intestine, the enteric-coated clothes are completely dissolved, and the encased Exenatide is released. The maximum blood concentration of Exenatide capsules can be reached after 5 hours of oral administration. This will improve compliance. However, this dosage form has not been listed in China.

The molecular structure of Liraglutide enables it to avoid being hydrolyzed by a DPP-4 enzyme with a half-life of about 13 hours. Liraglutide was approved internationally as a weight-loss drug in 2014. One experiment has shown that the weight effect persists for at least 12 months with the long-term use of Liraglutide in diabetics. This suggests that GLP-1 analogs have a relatively reliable long-term effect on obesity. But at the same time, other studies have shown that increased heart rate due to Liraglutide may be harmful to patients with severe heart failure. Therefore, further studies are needed to assess the long-term effects of Liraglutide.

Currently, GLP-1 analogs can be effective for obese patients through multiple targets, including the prevention and treatment of its complications. However, in China, GLP-1 has not been approved for use in patients with simple obesity. Therefore, clinical data are scarce, and only foreign clinical trials and animal tests are needed, lacking the experience of China's unique population. The therapeutic effects of GLP-1 on simple obesity need to be further explored.

A combination of some weight-loss drugs has been shown to be beneficial for weight loss, such as Phentermine and Lorcaserin [89]. At the same time, whether the combined application of GLP-1 with a variety of different mechanism drugs is beneficial to obese patients can be further studied.

Above all, obesity is a kind of more body fat content as the main characteristics of chronic diseases; GLP-1 and GLP-1 analogs treatment for obesity cannot only remove excess fat but also prevent the complications but for the role of long-term outcome is uncertain, still needs further research.

\section{References}

1. Lee PC, Dixon J (2017) Pharmacotherapy for obesity. Australian Family Physician 46: 472-477.

2. Curry SA (2017) Obesity epidemic: pharmaceutical weight loss. Rhode Island Medical Journal 100: 18.

3. China Obesity Working Group Data Summary Analysis Collaboration Group (2002) Predictive value of adult body mass index and waist circumference for abnormal risk factors of related diseases: suitable body mass index and waist circumference cut point study. Chin J Epid 23: 5-10.

4. Ye Z, Liu G, Guo J, Su Z (2018) Hypothalamic endoplasmic reticulum stress as a key mediator of obesity-induced leptin resistance. Obes Rev 19: 770-785.

5. Gadde KM, Pritham YR (2017) Pharmacotherapy of obesity: clinical trials to clinical practice. Curr Diab Rep 17: 34.

6. Progress in psychotropic drugs and psychotherapy for obesity.

7. Oen G, Kvilhaugsvik B, Eldal K, Halding AG (2018) Adolescents' perspectives on everyday life with obesity: a qualitative study. Int J Qual Stud Health Well-being 13: 1479581.

8. Antza C, Stabouli S, Natsis M, Doundoulakis I, Kotsis V (2017) Obesity-induced hypertension: New insights. Curr Pharm Des 23: 4620-4625.

9. Long-term weight loss maintenance.

10. Bhat SP, Sharma A (2017) Current drug targets in obesity pharmacotherapy-A review. Curr Drug Targets 18: 983-993.

11. Wilding JP (2017) Combination therapy for obesity. J Psychopharmacol 31: 1503-1508. 
12. Golden A (2017) Current pharmacotherapies for obesity: A practical perspective. J Am Assoc Nurse Pract 29: 43-54.

13. Roberts CA, Christiansen P, Halford JCG (2017) Tailoring pharmacotherapy to specific eating behaviours in obesity: Can recommendations for personalised therapy be made from the current data? Acta Diabetol 54: 715-725.

14. The past, present and future of weight loss drugs-reevaluation of anti-obesity drug safety.

15. Apovian CM, Aronne LJ, Bessesen DH, McDonnell ME, Murad MH, et al. (2015) Pharmacological management of obesity: an endocrine society clinical practice guideline. J Clin Endocrinol Metab 100: 342-62.

16. Sucharda $P$ (2017) Status of pharmacotherapy in current care of obese. Cas Lek Cesk 156: 319-322.

17. Hocking S, Dear A, Cowley MA (2017) Current and emerging pharmacotherapies for obesity in Australia. Obes Res Clin Pract 11: 501-521.

18. Roberts CA, Christiansen P, Halford JCG (2017) Tailoring pharmacotherapy to specific eating behaviours in obesity: Can recommendations for personalised therapy be made from the current data? Acta Diabetol 54: 715-25.

19. Kaplan LM (2010) Pharmacologic therapies for obesity. Gastroenterol Clin 39: 69-79.

20. Moldovan CP, Weldon AJ, Daher NS, Schneider LE, Bellinger DL, et al. (2016) Effects of a meal replacement system alone or in combination with phentermine on weight loss and food cravings. Obesity 24: 2344-2350.

21. Ryder JR, Kaizer A, Rudser KD, Gross A, Kelly AS, et al. (2005) Effect of phentermine on weight reduction in a pediatric weight management clinic. Int J Obes 41: 90-93.

22. Kim KK, Cho HJ, Kang HC, Youn BB, Lee KR (2006) Effects on weight reduction and safety of short-term phentermine administration in Korean obese people. Yonsei Med J 47: 614-25.

23. Reeve HL, Nelson DP, Archer SL, Weir EK (1999) Effects of fluoxetine, phentermine, and venlafaxine on pulmonary arterial pressure and electrophysiology. Am J Physiol Lung Cell Mol Physiol 276: 213-219.

24. Amphetamine tablet poisoning 1 case report Shi Chunhua (Jilin Integrated Traditional Chinese and Western Medicine Hospital, Jilin Jilin 132012).

25. Banks ML, Snyder RW, Fennell TR, Negus SS (2017) Role of damphetamine and d-methamphetamine as active metabolites of benzphetamine: Evidence from drug discrimination and pharmacokinetic studies in male rhesus monkeys. Pharmacol Biochem Behav 156: 30-38.

26. Cho AR, Yoon YH (2016) Central retinal vein occlusion noted 2 days after use of phendimetrazine as an appetite suppressant. JAMA Ophthalmol 134: 463-464.

27. Current status of obesity diagnosis and treatment

28. Chao AM, Wadden TA, Berkowitz RI (2018) The safety of pharmacologic treatment for pediatric obesity. Expert Opin Drug Saf 17: 379-385

29. Benaiges D, Pedro-Botet J, Flores-Le Roux JA, Climent E, Goday A (2017) Past, present and future of pharmacotherapy for obesity. Clin Investig Arterioscler 29: 256-264.

30. Sall D, Wang J, Rashkin M, Welch M, Droege C, et al. (2014) Orlistat-induced fulminant hepatic failure. Clin Obes 4: 342-347.
31. Martin CK, Redman LM, Zhang J, Sanchez M, Anderson CM, et al. (2011) Lorcaserin, a 5-HT2C receptor agonist, reduces body weight by decreasing energy intake without influencing energy expenditure. J Clin Endocrinol Metab 96: 837-845.

32. Oral treatment of obesity drugs- naltrexone/bupropion sustained release tablets.

33. Thomsen WJ, Grottick AJ, Menzaghi F, Reyes-Saldana H, Espitia S, et al. (2008) Lorcaserin, a novel selective human 5hydroxytryptamine $2 \mathrm{C}$ agonist: in vitro and in vivo pharmacological characterization. J Pharmacol Exp Ther 325: 577-587.

34. Kieffer TJ, Mclntosh CH, Pederson RA (1995) Degradation of glucose-dependent insulinotropic polypeptide and truncated glucagon-like peptide-1 in vitro and in vivo by dipeptidyl peptidase IV. Endocrinology 136: 3585-3596.

35. Aaboe K, Krarup T, Madsbad S, Holst JJ (2008) GLP-1: physiological effects and potential therapeutic applications. Diabetes Obes Metab 10: 994-1003.

36. Trujillo JM, Nuffer W (2014) GLP-1 receptor agonists for type 2 diabetes mellitus: recent developments and emerging agents. J Human Pharmcother Drug Ther 34: 1174-1186.

37. Pereira MJ, Lundkvist P, Kamble PG, Lau J, Martins JG, et al. (2018) A randomized controlled trial of dapagliflozin plus once-weekly exenatide versus placebo in individuals with obesity and without diabetes: metabolic effects and markers associated with bodyweight loss. Diabetes Ther 1: 1-22.

38. Kanoski SE, Fortin SM, Arnold M, Grill HJ, Hayes MR (2011) Peripheral and central GLP-1 receptor populations mediate the anorectic effects of peripherally administered GLP-1 receptor agonists, liraglutide and exendin-4. Endocrinology 152: 3103-3112.

39. Meeran K, O'shea D, Edwards CM, Turton MD, Heath MM, et al. (1999) Repeated intracerebroventricular administration of glucagon-like peptide-1-(7-36) amide or exendin-(9-39) alters body weight in the rat. Endocrinology 140: 244-250.

40. Holst JJ (1994) Glucagons-like peptide-1 (GLP-1) a newly discovered GI hormone. GI 107: 1948-1955.

41. Meier JJ, Gallwitz B, Salmen S, Goetze O, Holst JJ, et al. (2003) Normalization of glucose concentrations and deceleration of gastric emptying after solid meals during intravenous glucagonlike peptide- 1 in patients with type 2 diabetes. J Clin Endocrinol Metab 88: 2719-2725.

42. Maciel MG, Beserra BT, Oliveira FC, Ribeiro CM, Coelho MS, et al. (2018) The effect of glucagon-like peptide-1 and glucagon-like peptide-1 receptor agonists on energy expenditure: A systematic review and meta-analysis. Diabetes Res Clin Pract 142: 222-235.

43. Clinical efficacy of glucagon-like peptide-1 receptor agonist in the treatment of type 2 diabetes and safety research progress.

44. Sha S, Rahman CS, Chen Li, He Qin, Zhang Jing, et al. (2016) Effects of liraglutide on leptin, fat distribution and content in obese mice induced by high fat. Journal of Shandong University 54: 22-27.

45. Meier JJ, Gallwitz B, Salmen S, Goetze O, Holst JJ, et al. (2003) Normalization of glucose concentrations and deceleration of gastric emptying after solid meals during intravenous glucagonlike peptide- 1 in patients with type 2 diabetes. J Clin Endocrinol Metab 88: 2719-2725.

46. Buse JB, Rosenstock J, Sesti G, Schmidt WE, Montanya E, et al. (2009) Liraglutide once a day versus exenatide twice a day for type 2 diabetes: a 26-week randomised, parallel-group, multinational, open-label trial (LEAD-6). Lancet 374: 39-47. 
47. Mehta A, Marso SP, Neeland IJ (2017) Liraglutide for weight management: a critical review of the evidence. Obes Sci Pract 3: 3-14.

48. Iepsen EW, Zhang J, Thomsen HS, Hansen EL, Hollensted M, et al. (2018) Patients with obesity caused by melanocortin-4 receptor mutations can be treated with a glucagon-like peptide- 1 receptor agonist. Cell Metab 28: 23-32.

49. Effects of liraglutide on body weight, blood sugar, blood lipid and liver function in obese mice induced by high fat diet.

50. Mikhail N (2018) Cardiovascular effects of liraglutide. Current Hypertension Reviews.

51. Davies MJ, Aronne LJ, Caterson ID, Thomsen AB, Jacobsen PB (2018) Liraglutide and cardiovascular outcomes in adults with overweight or obesity: A post hoc analysis from SCALE randomized controlled trials. Diabetes Obes Metab 20: 734-739.

52. Kaneko M, Narukawa M (2018) Assessment of cardiovascular risk with glucagon-like peptide-1 receptor agonists in patients with type 2 diabetes using an alternative measure to the hazard ratio. Ann Pharmacother 52: 632-638.

53. Vrang N, Jelsing J, Simonsen L, Jensen AE, Thorup I, et al. (2012) The effects of $13 \mathrm{wk}$ of liraglutide treatment on endocrine and exocrine pancreas in male and female ZDF rats: a quantitative and qualitative analysis revealing no evidence of drug-induced pancreatitis. Am J Physiol Endocrinol Metab 303: 253-264.

54. Advances in studies on structure and function of GLP-1 analogue proteins.

55. Voronova V, Zhudenkov K, Penland RC, Boulton DW, Helmlinger G, et al. (2018) Exenatide effects on gastric emptying rate and the glucose rate of appearance in plasma: A quantitative assessment using an integrative systems pharmacology model. Diabetes Obes Metab 20: 2034-2038.

56. Hu Y, Liu J, Wang G, Xu Y (2018) The effects of exenatide and metformin on endothelial function in newly diagnosed type 2 diabetes mellitus patients: a case-control study. Diabetes Ther 9: 1295-1305.

57. Andersen A, Lund A, Knop FK, Vilsboll T (2018) Glucagon-like peptide-1 in health and disease. Nat Rev Endocrinol 14: 390-403.

58. Owens DR, Monnier L, Bolli GB (2013) Differential effects of GLP-1 receptor agonists on components of dysglycaemia in individuals with type 2 diabetes mellitus. Diab Metab 39: 485-496.

59. Guja C, Frias JP, Somogyi A, Jabbour S, Wang H, et al. (2018) Effect of exenatide QW or placebo, both added to titrated insulin glargine, in uncontrolled type 2 diabetes: The duration-7 randomized study. Diabetes Obes Metab 20: 1602-1614.

60. Ahrén B, Atkin SL, Charpentier G, Warren ML, Wilding JPH, et al. (2018) Semaglutide induces weight loss in subjects with type 2 diabetes regardless of baseline BMI or gastrointestinal adverse events in the sustain 1 to 5 trials. Diabetes Obes Metab 20: 2210-2219.

61. Bekiari E, Karagiannis T, Tsapas A (2018) In type 2 diabetes, weekly semaglutide reduced $\mathrm{HbA} 1 \mathrm{c}$ and increased weight loss more than weekly exenatide ER. Ann Intern Med 168: JC46.

62. Andreadis P, Karagiannis T, Malandris K, Avgerinos I, Liakos A, et al. (2018) Semaglutide for type 2 diabetes mellitus: A systematic review and meta-analysis. Diabetes Obes Metab 20: 2255-2263.

63. Korsatko S, Jensen L, Brunner M, Sach-Friedl S, Tarp MD, et al. (2018) Effect of once-weekly semaglutide on the counterregulatory response to hypoglycaemia in subjects with type 2 diabetes: a randomised, placebo-controlled, double-blind, crossover trial. Diabetes Obes Metab 20: 2565-2573.

64. Warren M, Chaykin L, Trachtenbarg D, Nayak G, Wijayasinghe N, et al. (2018) Semaglutide as a therapeutic option for elderly patients with type 2 diabetes: Pooled analysis of the sustain 1-5 trials. Diabetes Obes Metab 20: 2291-2297.

65. Abdel-Latif RG, Heeba GH, Taye A, Khalifa MMA (2018) Lixisenatide, a novel GLP-1 analog, protects against cerebral ischemia/reperfusion injury in diabetic rats. Naunyn Schmiedebergs Arch Pharmacol 391: 705-717.

66. Rosenstock J, Raccah D, Korányi L, Maffei L, Boka G, et al. (2013) Efficacy and safety of lixisenatide once daily versus exenatide twice daily in type 2 diabetes inadequately controlled on metformin: a 24-week, randomized, open-label, active-controlled study (GetGoal-X). Diab Care 36: 2945-2951.

67. Madsbad S (2016) Review of head-to-head comparisons of glucagon-like peptide-1 receptor agonists. Diabetes Obes Metab 18: 317-332.

68. Fonseca VA, Alvarado-Ruiz R, Raccah D, Boka G, Miossec $P$, et al. (2012) Efficacy and safety of the once-daily GLP-1 receptor agonist lixisenatide in monotherapy:a randomized, double-blind, placebocontrolled trial in patients with type 2 diabetes (GetGoalMono). Diab Care 35: 1225-1231.

69. Kapitza C, Forst T, Coester HV, Poitiers F, Ruus P, et al. (2013) Pharmacodynamic characteristics of lixisenatide once daily versus liraglutide once daily in patients with type 2 diabetes insufficiently controlled on metformin. Diabetes Obes Metab 15: 642-649.

70. Monami M, Nreu B, Scatena A, Cresci B, Andreozzi F, et al. (2017) Safety issues with glucagon-like peptide-1 receptor agonists (pancreatitis, pancreatic cancer and cholelithiasis): Data from randomized controlled trials. Diabetes Obes Metab 19: 1233-1241.

71. Chis BA, Fodor D (2018) Acute pancreatitis during GLP-1 receptor agonist treatment. A case report. Clujul Med 91: 117-119.

72. Consoli A, Formoso G (2015) Potential side effects to GLP-1 agonists: Understanding their safety and tolerability. Expert Opin Drug Saf 14: 207-218.

73. Ballav C, Gough S (2012) Bydureon: long-acting exenatide for once-weekly injection. Prescriber 23: 30-33.

74. Scott LJ (2012) Exenatide extended-release: a review of its use in type 2 diabetes mellitus. Drugs 72: 1679-1707.

75. Liancheng Z, Yangfeng W, Ying L, Beifan Z, Jun Y. Relationship between different body mass index and waist circumference level and other cardiovascular risk factors.

76. Goud A, Zhong J, Peters M, Brook RD, Rajagopalan S (2016) GLP-1 agonists and blood pressure: $A$ review of the evidence. Curr Hypertens Rep 18: 16.

77. Kim M, Platt MJ, Shibasaki T, Quaggin SE, Backx PH, et al. (2013) GLP-1 receptor activation and Epac2 link atrial natriuretic peptide secretion to control of blood pressure. Nat Med 19: 567.

78. Chang G, Zhang D, Yu H, Zhang P, Wang Y, et al. (2013) Cardioprotective effects of exenatide against oxidative stressinduced injury. International journal of molecular medicine. Int J Mol Med 32: 1011-1020.

79. Ussher JR, Drucker DJ (2012) Cardiovascular biology of the incretin system. Endocr Rev 33: 187-215.

80. Kimura T, Obata A, Shimoda M, Shimizu I, da Silva Xavier G, et al. (2018) Down-regulation of vascular GLP-1 receptor expression in human subjects with obesity. Sci Rep 8: 10644. 
81. Xiaoyun S GLP-1 and type 2 diabetes fatty liver and clinical management.

82. Khoo J, Hsiang J, Taneja R, Law NM, Ang TL (2017) Comparative effects of liraglutide $3 \mathrm{mg}$ vs structured lifestyle modification on body weight, liver fat and liver function in obese patients with non-alcoholic fatty liver disease: A pilot randomized trial. Diabetes Obes Metab 19: 1814-1817.

83. Lynch L, Hogan AE, Duquette D, Lester C, Banks A, et al. (2016) iNKT cells induce FGF21 for thermogenesis and are required for maximal weight loss in GLP1 therapy. Cell Metab 24: 510-519.

84. Fei Z, Yuxi W. The effect of obesity on immune function.

85. Effects of liraglutide on lipid metabolism, bone metabolism and inflammatory factors in obese overweight type 2 diabetic patients.
86. Immunomodulatory effects and mechanisms of glucagon-like peptide-1.

87. Bhushan R, Elkind-Hirsch KE, Bhushan M, Butler WJ, Duncan K, et al (2008) Exenatide use in the management of metabolic syndrome: a retrospective database study. Endocr Pract 14: 993-999.

88. Nguyen HN, Wey SP, Juang JH, Sonaje K, Ho YC, et al. (2013) The glucose-lowering potential of exendin- 4 orally delivered via a $\mathrm{pH}-$ sensitive nanoparticle vehicle and effects on subsequent insulin secretion in vivo. Biomaterials 32: 2673-2682.

89. Rebello CJ, Nikonova EV, Zhou S, Aronne LJ, Fujioka K, et al. (2018) Effect of Lorcaserin alone and in combination with phentermine on food cravings after 12-week treatment: A randomized substudy. Obesity 26: 332-339. 